J. Lake Sci. (湖泊科学), 2012, 24(3):355-361

http: //www. jlakes.org. E-mail : jlakes@niglas.ac.cn

(C) 2012 by Journal of Lake Sciences

\title{
沉水植物驱动的水环境钙蜃与水体磷循环的关系"
}

\author{
朱端卫, 朱 红, 倪玲珊, 肖鹏程 \\ (华中农业大学资源与环境学院植物营养与生态环境研究室,武汉 430070)
}

\begin{abstract}
摘 要: 水体磷循环是水柱和对应的沉积物中发生的各种非生物和生物的磷迁移转化过程. 与此同时, 沉积物中䥻通过 沉水植物吸收和转运, 从该类植物的叶面释放至水柱中, 释放的 $\mathrm{Ca}^{2+}$ 与水柱中的 $\mathrm{CO}_{3}^{2-}$ 一起形成碳酸钙. 在这一过程中, 水柱中少量溶解性磷分配在碳酸钙中形成 $\mathrm{CaCO}_{3}-\mathrm{P}$ 共沉淀, 导致水体中可溶性磷向难溶性磷转化, 这种由沉水植物驱动 的水环境钙泵在水体磷循环中发挥着重要作用. 研究证明,沉水植物䓚草叶面上有 $\mathrm{CaCO}_{3}-\mathrm{P}$ 共沉淀的形成,且这种共沉 淀的含磷量变化范围很宽. 另一方面, 新近沉积物中钙与磷的沉淀物存在一个由聚磷酸盐向磷灰石逐渐演变过程, 而沉 水植物叶面上的含磷共沉淀作用是否也存在由聚磷酸盐向磷灰石的变质过程,该过程在沉水植物生长期间是否发生关 系到沉水植物除磷效果值得深人研究. 本文从水体磷循环概述、钻在水体磷循环中的作用和沉水植物驱动的水环境钙泵 假说及其在水体磷循环中的意义等方面综述了钙在水环境中的迁移对水体磷循环的贡献.
\end{abstract}

关键词: 沉水植物; 钲原; $\mathrm{CaCO}_{3}-\mathrm{P}$ 共沉淀; 上覆水;沉积物; 磷循环

\section{Relationship between calcium pump driven by submerged macrophytes and phosphorus cycle in water}

\author{
ZHU Duanwei, ZHU Hong, NI Lingshan \& XIAO Pengcheng \\ (Laboratory of Plant Nutrition and Ecological Environment Research, Huazhong Agricultural University, Wuhan 430070, P. \\ R. China)
}

\begin{abstract}
Phosphorus cycle in water contains processes of various abiotic and biotic transformations of phosphorus in water column and corresponding sediments. Calcium in the sediment can be up-taken by submerged macrophytes and then released to the water column from the leaves, which can form calcium carbonate with $\mathrm{CO}_{3}^{2-}$ in the water column. In this process, a small amount of soluble phosphorus incorporated into calcium carbonate forming $\mathrm{CaCO}_{3}-\mathrm{P}$ coprecipitation, leading to the transformation of soluble phosphorus to hard dissolved phosphorus. This kind of calcium pump driven by submerged macrophytes in water environment plays an important role in the phosphorus cycle. Research proves that $\mathrm{CaCO}_{3}-\mathrm{P}$ coprecipitation can be formed on the leave surface of submerged macrophyte Potamogeton crispus and the content of total phosphorus in the coprecipitation changes in a wide range. In addition, the coprecipitation of calcium and phosphorus in the newly-formed sediments exists a process that polyphosphate can evolve to apatite gradually. It is not clear if this process occurred during the coprecipitation of calcium and phosphorus on the leave surface of submerged macrophytes is related to the phosphorus removal by submerged macrophytes. In this review, the research progress on the contributions of calcium transportation to the phosphorus cycle in the water system was introduced in detail, together with phosphorus cycle, function of calcium, and calcium pump hypothesis driven by submerged macrophytes and its implications.
\end{abstract}

Keywords: Submerged macrophytes; calcium pump; $\mathrm{CaCO}_{3}-\mathrm{P}$ coprecipitation; overlying water; sediment; phosphorus cycle

在世界范围内分布的部分浅水湖泊担负着调节全球内陆气候及水量、维系淡水生物繁衍及其多样性和 为人畜提供饮用水源等重要的生态功能. 随着人类社会的快速发展, 水体富营养化过程日益加剧, 浅水湖泊 的生态功能日渐脆弱. 磷是所有生命活动的必需元素, 水体磷的生物地球化学循环失衡是湖泊发生生态问 题的重要原因之一 ${ }^{[1]}$. 沉水植物其根系扎在沉积物中而枝条生长在上覆水中, 其去除水体中氮、磷的效率占

* 国家自然科学基金项目 (40973056) 和教育部博士点基金项目 (20100146110020) 联合资助. $2011-09$ - 15 收稿; 2011-11-28 收修改稿. 朱端卫,男,1956 年生, 教授;E-mail:zhudw@ mail. hzau. edu. cn. 
4 类大型水生植物总和的 $40 \%{ }^{[2]}$, 其净水作用在高等水生植物中首当其冲, 展现了其对富营养化水体水质 修复的前景 ${ }^{[3]}$. 浅水湖泊生态链中的沉水植物是怎样去除水体营养物质乃至对磷的生物地球化学循环产生 深刻影响是湖沼学、地球化学和环境化学共同面临的科学问题, 回答这一问题可为解决水体磷生物地球化 学循环失衡问题提供理论依据,对富营养化水体的生态重建和浅水湖泊的生态保护具有学术和现实意义.

\section{1 水体磷循环概述}

水体磷循环是水柱中和对应的沉积物中发生的各种非生物和生物的磷迁移过程 ${ }^{[4]}$. 总体来说, 水体流 动和扩散是造成水体磷迁移的宏观动力, 如流经水体植被的水流会引起植被下方沉积物中磷的释放 ${ }^{[5]}$. 发 生在沉积物中粘土和金属氧化物上的磷吸附/解吸是水体磷转化主要的非生物微观过程,其中磷在铁、铝氧 化物的 $\mathrm{Me}-\mathrm{OH}_{2}^{+}$和 $\mathrm{Me}-\mathrm{OH}$ 位点上的配位体交换是这一过程的主要机制 ${ }^{\left[{ }^{[6]}\right.}$. 沉积物表面形成的氧化层可以通 过间隙水从上覆水中吸附溶解态活性磷 $(S R P)^{[7]}$. 氧化性间隙水中存在的磷酸铁就是水合氧化铁与被吸附 的磷进一步反应的结果; 在厌氧条件下,间隙水中高浓度 SRP 在丰富的有机质作用下形成亚铁磷酸盐矿物 即蓝铁矿 ${ }^{[8]}$. 从这个意义上讲, 磷在金属氧化物上的吸附只是磷转化过程中的一种过渡态. 底栖附着生物和 水中浮游植物可以吸收有机和无机磷组分, 这样既可以从水柱中除去磷, 也可以阻止磷由沉积物向水柱中 扩散 ${ }^{[9]}$. 细菌和真菌通过代谢作用、改变沉积物表面的氧化还原条件或产生一些复杂的有机磷中间体来调 节沉积物一水界面之间磷的通量以对水体磷循环施加影响 ${ }^{[10]}$.

高等水生植物是水体生态系统中不可或缺的组成部分. 目前,其与水体磷循环有关的研究结果主要有: 在生长季节吸收磷, 死亡后释放出 SRP; 减缓水流速度, 增强包括不同颗粒有机物在内的沉积物对磷的吸 附; 给附着生物增加附着面积, 为水生动物提供生存条件以加强上覆水中的磷向生物体转移 ${ }^{[4]}$. 从上述结果 中不难看出, 针对水生植物参与水体磷循环的功能和效果已有一定的研究, 但从理论上认识水生植物生长 与水体磷循环的关系还缺乏系统性研究.

\section{2 钙在水体磷循环中的作用}

\section{1 水中 $\mathrm{CaCO}_{3}-\mathrm{P}$ 共沉淀的形成}

众所周知,水体磷循环的研究非常注重沉积物磷的分级及其与水质的关系 ${ }^{[11]}$. 然而, 何种磷形态更能 控制水中磷浓度这个问题始终未能得到很好的回答. 研究表明, 氧化条件可促进磷在氧化铁/氢氧化铁上的 吸附或形成铁磷沉淀,但水柱中 SRP 仍可达到 $0.1 \mathrm{mg} / \mathrm{L}$, 而在 $\mathrm{CaCO}_{3}-\mathrm{P}$ 共沉淀条件下, SRP 可下降到远低于 $0.1 \mathrm{mg} / \mathrm{L}$ 的水平 ${ }^{[12-13]}$. 海洋磷的生物地球化学循环与微生物或硅藻类之间关系密切 ${ }^{[14]}$, 其中钲是维持海洋 磷库,控制磷从海底返回水中的主要机制. 如硫细菌 Thiomargarita namibiensis 吸收来自好氧水柱中的磷酸 盐, 然后通过氧化沉积物中硫化物获取能量的同时释放出磷酸盐, XRD 分析表明,这些磷酸盐在钲的作用下 转化成差基磷灰石 ${ }^{[15]}$.

发生在水柱中的沉淀往往需有生物膜 (如藻类) 作为悬浮的成核位点 ${ }^{[16]}$, 在结晶增长过程中, 可溶性磷 酸盐可以与 $\mathrm{CaCO}_{3}$ 发生共沉淀 ${ }^{[17-18]}$, 经测定, 所得共沉淀中磷钙比一般为 $2 \sim 6 \mathrm{mg} \mathrm{P} / \mathrm{g} \mathrm{Ca}^{[17]} \cdot \mathrm{CO}_{2}$ 在光合作 用下被沉水植物吸收, 从而引起上覆水 $\mathrm{pH}$ 值升高. 从生物化学的角度考察这一过程为 $2 \mathrm{HCO}_{3}^{-} \rightarrow \mathrm{CH}_{2} \mathrm{O}+\mathrm{O}_{2}$ $+\mathrm{CO}_{3}^{2-}$, 这一反应导致上覆水碱度增加 $\left(\mathrm{CO}_{3}^{2-}\right.$ 增加是其主要原因 $), \mathrm{CaCO}_{3}$ 过饱和而发生 $\mathrm{CaCO}_{3}$ 沉淀 ${ }^{[19]}$, 同 时, 在这种高 $\mathrm{pH}$ 环境中磷酸盐和 $\mathrm{Ca}^{2+}$ 的沉淀溶解度进一步减少, 接着就发生 $\mathrm{CaCO}_{3}-\mathrm{P}$ 的共沉淀 ${ }^{[20]}$. 通过微 探针对分布在沉积物表层薄片中方解石矿物进行观察后发现,源于生物性的方解石形成共沉淀更为有效, 能起到固定 P、 Fe 和 $\mathrm{Mn}$ 的作用 ${ }^{[21-22]}$. 用 X 射线苂光能谱分析还发现了这些富磷微域中磷形态的变质,这一 过程先生成聚磷酸盐, 然后磷和钲形成过渡态结晶体,最后结晶体向磷灰石深度转化 ${ }^{[23]}$.

\section{2 沉水植物叶面 $\mathrm{CaCO}_{3}-\mathrm{P}$ 共沉淀现象}

在湖泊碳收支平衡的研究中发现,夏季沉水植物生长旺盛,上覆水中碱度下降,大量的碳酸根在形成碳 酸钻的过程中使湖泊碱度下降 $15 \% \sim 25 \%{ }^{[19]}$. 用南湖上覆水进行的盆栽实验表明,未种植菹草的上覆水 TP 为 $0.56 \pm 0.03 \mathrm{mg} / \mathrm{L}$, 种植菹草的上覆水 TP 下降到 $0.23 \pm 0.01 \mathrm{mg} / \mathrm{L}$, 下降 $59 \%$, 如果在沉积物中新增一定

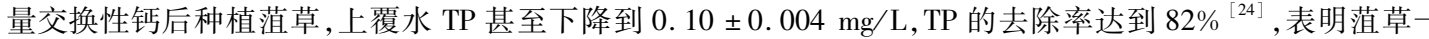


钲一上覆水 TP 之间关系密切. 在一些浅水湖泊中生长的沉水植物菹草叶面产生大量白色颗粒物质可能是由 于水体中钙盐的淀积, 菹草以其叶面承接了这类淀积物. 在实验室用湖泊沉积物培养菹草几周后, 菹草叶片 上有白色颗粒物存在, 该颗粒物经 $\mathrm{HCl}$ 溶解后测得其磷钻比为 $2.2 \sim 3.4 \mathrm{mg} \mathrm{P} / \mathrm{g} \mathrm{Ca}$, 与有关文献介绍溶解性 磷酸盐同方解石 $\left(\mathrm{CaCO}_{3}\right)$ 发生共沉淀的磷钲比例相符 ${ }^{[17-18]}$, 如在 Veluwe 湖泊中, 上覆水中磷的初始值在 $0.1 \sim 0.2 \mathrm{mg} / \mathrm{L}$ 条件下, $\mathrm{CaCO}_{3}-\mathrm{P}$ 共沉淀的 $\mathrm{P}: \mathrm{Ca}$ 为 $2.7 \sim 7.2 \mathrm{mg} \mathrm{P} / \mathrm{g} \mathrm{Ca}^{[17]}$. 我们用武昌南湖沉积物以含磷量 较高 $(1.0 \mathrm{mg} / \mathrm{L} \mathrm{P})$ 的上覆水培养菹草甚至在其叶面可以得到含磷量更高的淀积物 (表 1 ). 上述研究表明, 在 沉水植物生长与水体磷的循环或水柱中磷的迁移关系中, 钻起着不容忽视的作用.

表 1 菹草叶面淀积物钲磷比例

Tab. 1 The ratio of calcium and phosphorus in the precipitates on leaves

\begin{tabular}{|c|c|c|c|c|c|c|}
\hline \multirow{2}{*}{ 菹草编号 } & \multicolumn{3}{|c|}{ 超声振荡提取(先) } & \multicolumn{3}{|c|}{$1 \mathrm{~mol} / \mathrm{L} \mathrm{HCl}$ 溶解提取 (后) } \\
\hline & $\mathrm{P} / \mathrm{mg}$ & $\mathrm{Ca} / \mathrm{mg}$ & $\mathrm{Ca} / \mathrm{P} /(\mathrm{mg} / \mathrm{mg})$ & $\mathrm{P} / \mathrm{mg}$ & $\mathrm{Ca} / \mathrm{mg}$ & $\mathrm{Ca} / \mathrm{P} /(\mathrm{mg} / \mathrm{mg})$ \\
\hline 1 & 0.202 & 0.136 & 0.67 & 0.153 & 0.326 & 2.12 \\
\hline 2 & 0.172 & 0.223 & 1.29 & 0.084 & 0.197 & 2.34 \\
\hline 3 & 0.176 & 0.031 & 0.18 & 0.062 & 0.141 & 2.28 \\
\hline
\end{tabular}

用 EM-XPS 观察到菹草叶面上白色微晶体状物质 (图 1). 淀积物基本形貌呈针状, 以簇状态存在 (图 1a), 基本上是方解石结构 ${ }^{[25]}$, 在一些更细的块状结构中发现有较多的磷存在 (图 $1 \mathrm{~b}$ ). 其中针状矿物中 $\mathrm{C} 、 O$ 和 $\mathrm{Ca}$ 的原子百分比约为 38:55:6, 颗粒状矿物中 $\mathrm{C} 、 \mathrm{O}$ 和 $\mathrm{Ca}$ 的原子百分比约为 $25: 61: 12$. 已有实验表明,沉 水植物能够使底质中活性磷转化为活性较小的钲结合态磷沉积于底质中 ${ }^{[26]}$, 但沉水植物的生长是否从叶片 表面释放出䥻离子, 从而证实这一过程中 $\mathrm{CaCO}_{3}-\mathrm{P}$ 共沉淀生成仅在我们实验室进行了初步研究(见 3.2 节). 另一方面, 从 Diaz 等 ${ }^{[23]}$ 的研究结果可以看出, 新近沉积物中钲与磷的沉淀物存在一个由聚磷酸盐向磷 灰石逐渐演变的过程. 而本实验发现的沉水植物叶面上含磷共沉淀, 是否也存在由聚磷酸盐向磷灰石的变 质过程,关系到沉水植物除磷效果, 值得深人研究.
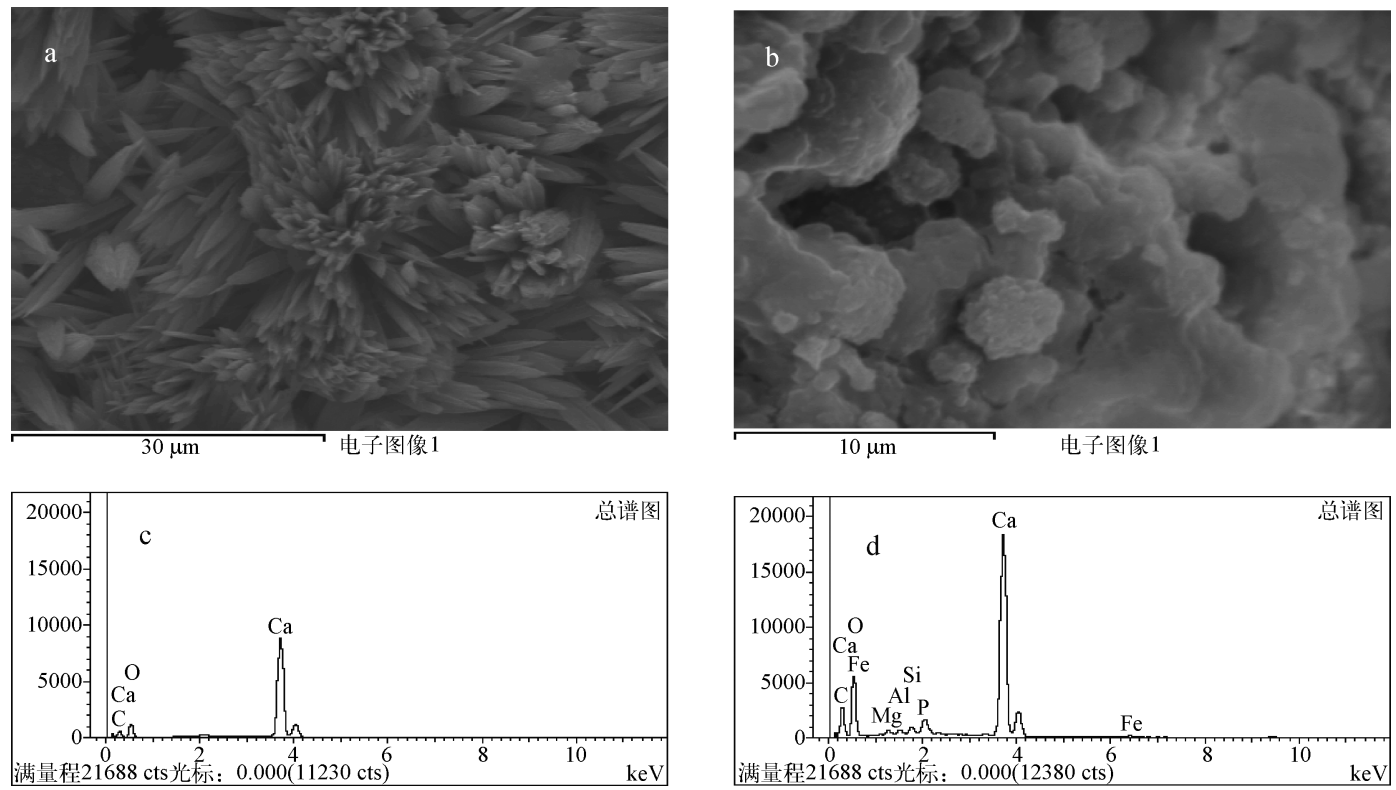

图 1 菹草叶片表面淀积物 SEM 照片 $(a 、 b)$ 和对应 XPS 图谱 $(c 、 d)$

Fig. 1 SEM images of precipitates $(a, b)$ in the experiment and corresponding XPS spectras $(c, d)$ 


\section{3 沉水植物驱动的水环境钙洜假说及其在水体磷循环中的意义}

洜假说已深人到多种学说当中, 并合理解释了众多自然现象. 总体来说, 物质的泵假说是物质运转的途 径和能量来源之集合,物质经过生物的作用而发生的迁移过程可用“生物泵”来描述 ${ }^{[27-28]}$.

\section{1 植物对钙的转运}

钙是植物必需的营养元素, 在植物生长发育和应对环境胁迫中处于中心调控地位 ${ }^{[29]}$. Ca ${ }^{2+}$ 进人植物根 系细胞, 在根系中进行横向短距离运输进人木质部, 其吸收主要发生在尚未形成凯氏带的根尖和侧根形成 部位 $^{[30]}$, 这一运输特点可以在沉水植物的根系特征中得到充分体现, 包括沉水植物在内的湿生植物具有发 达的须根 ${ }^{[31]}$, 这种须根又细又长, 根尖分生组织非常发达 ${ }^{[32]}$. 现已发现, 部分 $\mathrm{Ca}^{2+}$ 还可以由此通过离子通道 流进内皮层细胞而转人共质体并到达木质部薄壁细胞组织中 ${ }^{[33-34]}$. 在水体生态系统中, 水生植物对钙的吸 收尚未见报道, 但可以想象, 这一过程与沉积物中钲的活性或难溶态钲形态的活化有关, 植物根系在吸收营 养的同时,其分泌物也可能加快沉积物中 $\mathrm{CaCO}_{3}-\mathrm{P}$ 共沉淀中磷向更难溶的铁磷转化, 释放出的部分活性钲 被沉水植物吸收, 从而加速沉积物钲向水柱中的迁移.

目前,除根系吸收外,涉及对钙由植物向环境中迁移的研究主要集中在探讨陆生植物盐分分泌机理. 盐 土植物 Reaumuria hirtella Jaub. 在盐分分泌中, $\mathrm{Ca}^{2+}$ 的分泌比例在 $\mathrm{Na}^{+}$之后占据第二位 ${ }^{[35]}$. X 射线微区分析 结果表明,獐茅的盐腺主要分布在叶脉附近, 可以有效地将包括 $\mathrm{Ca}^{2+}$ 在内的盐分从表皮细胞和叶肉细胞转 运到基细胞, 再由帽细胞分泌到植株体外 ${ }^{[36]}$. 上述发现为研究沉水植物中的钙由地上部向水柱中迁移过程 提供了借鉴.

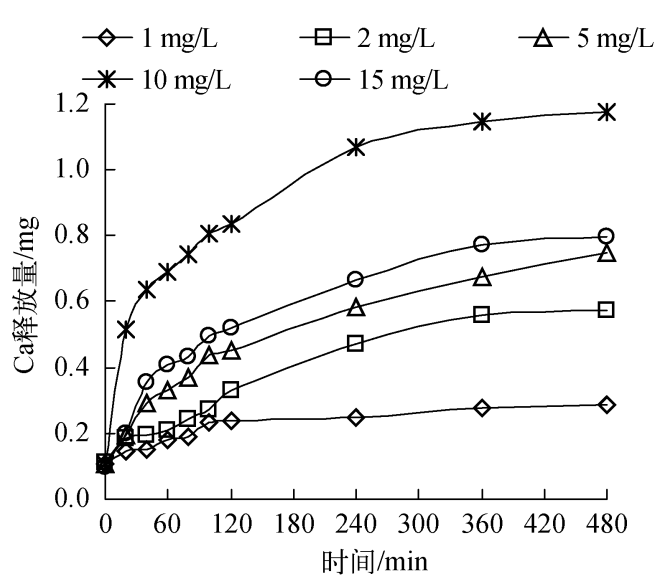

图 2 菹草茎叶培养过程中叶面向上覆水中钻释放 随时间的变化曲线

(其中 $1 \sim 15 \mathrm{mg} / \mathrm{L}$ 为向菹草根系的供钲水平)

Fig. 2 The change of total calcium released from leaf of Potamogeton crispus to overlying water under culture process at different times

对沉水植物䓚草根系吸收钙后其叶片释放钙的初 步研究表明, 在 $0 \sim 10 \mathrm{mg} / \mathrm{L}$ 范围内, 茎叶分泌的钙量 在相同的时间段随根培养液中钙离子浓度的升高而增 加,但当根培养液中 $\mathrm{Ca}^{2+}$ 浓度过高时茎叶钙的分泌量 下降, 如根培养液中 $\mathrm{Ca}^{2+}$ 浓度为 $15 \mathrm{mg} / \mathrm{L}$ 时, 菹草茎叶 钙的分泌量反而小于根培养液中 $\mathrm{Ca}^{2+}$ 浓度为 $10 \mathrm{mg} / \mathrm{L}$ 时的分泌量(图 2).

\section{2 生物膜钙泵的运行机制及作用}

在微观上, 生物膜上的钾洜、质子洜等假说解释了 动植物细胞间钾离子逆浓度转运的能量来源. 同样, 钙 在生物体内、生物体与环境之间迁移的能量供给及钙 离子在细胞水平上转运过程可藉 $\mathrm{Ca}^{2+}$-ATP 酶 $\left(\mathrm{Ca}^{2+}\right.$ ATPase) 的作用即钙洜 (calcium pump) 的机制加以阐 明 ${ }^{[37-38]} \cdot \mathrm{Ca}^{2+}$-ATPase 存在两种构像状态, 即对 $\mathrm{Ca}^{2+}$ 亲 和性高的 $\mathrm{E} 1$ 态和对 $\mathrm{Ca}^{2+}$ 亲和性低的 $\mathrm{E} 2$ 态. $\mathrm{E} 1$ 和 $\mathrm{E} 2$ 通过与 $\mathrm{Ca}^{2+}$ 的结合及释放, 进行能量的循环 ${ }^{[39]}$; 也有 报道认为, 尽管 $\mathrm{Ca}^{2+}$-ATPase 对 $\mathrm{Ca}^{2+}$ 有较高的亲合性 $\left(K_{\mathrm{m}}=0.2 \sim 1.0 \mu \mathrm{mol} / \mathrm{L}\right)$, 但其运输 $\mathrm{Ca}^{2+}$ 的能力 低 ${ }^{[00-41]}$. 近年来, $\mathrm{Ca}^{2+}$-ATP 酶作用机理更加明确, 其核 苷上进行着 $\mathrm{Ca}^{2+}$ 离子的结合和转运, 该酶的磷酸化引 发了其在生物膜上的构象变化, 导致 $\mathrm{Ca}^{2+}$ 离子跨膜运输相继形成, 6 种中间体中的第 4 个中间体的变构促进 了 $\mathrm{Ca}^{2+}$ 的释放 ${ }^{[38]}$. 有关专家对此动力学过程进行了数学模拟 ${ }^{[42]}$.

$\mathrm{Ca}^{2+}$-ATPase 存在于植物几乎所有细胞膜上,包括质膜、液泡膜、内质网膜、线粒体膜、质体和叶绿体膜 以及核膜等 ${ }^{[37]}$. 在植物根中, 䥻由木质部薄壁细胞组织进人中柱质外体可能需要 $\mathrm{Ca}^{2+}-\mathrm{ATPase}$ 的驱动, 根系 维管束组织钻素吸收可能通过共质体 (胞间连丝) 和质外体两种途径, 而钙离子通道、 $\mathrm{Ca}^{2+}-\mathrm{ATPa}$ e 和 $\mathrm{Ca}^{2+} / \mathrm{H}^{+}$反向转运蛋白等可能参与了根系细胞对钙的吸收 ${ }^{[30]}$. 


\section{3 水环境钙洜假设}

与生物膜上的洜假说相对应, 研究者为了从微观生态学的角度解释水体中溶解性无机碳产生的过程, 将海洋溶解性无机碳来源动力用海洋表层有机碳/碳酸钲洜假说来描述 ${ }^{[14]}$. 如前所述, 溶解性磷酸盐同方解 石 $\left(\mathrm{CaCO}_{3}\right)$ 一道形成共沉淀过程广泛存在于自然水体中, 而附着生物和藻类的光合作用可为其由水柱中吸 收磷提供有利条件, 如水中生物膜物质白天对 $\mathrm{CO}_{2}$ 的吸收使水柱局部 $\mathrm{pH}$ 显著升高, 为沉淀的成核作用提供 了能量, 促进微域 $\mathrm{CaCO}_{3}-\mathrm{P}$ 共沉淀形成 ${ }^{[12,43]}$. 另一方面研究表明, 作为浅水湖泊中的物种, 沉水植物在水体 $\mathrm{Ca}^{2+}$ 迁移中作用非常明显, 如饥饿后的菹草根系最初吸收䥻的米氏常数为 $2.57 \mathrm{mmol} / \mathrm{L}$, 比杨洪强等 ${ }^{[44]}$ 得 出的相同温度下苹果砧木吸收钲的米氏常数高 2 个数量级, 且这一沉水植物在水柱中对应的钙最大释放量 可达到 $61.5 \mathrm{mmol} / \mathrm{kg}$.

很显然, 沉水植物对钻如此强烈的吸收与释放, 不仅见证了促 使钙在植物体与环境之间转移的植物组织的能量水平 (ATP), 有关 菹草吸收和释放钙的动力学实验结果 (图 2) 初步预示着钻由沉水 植物茎叶向水柱迁移通道的存在,尽管对其在水体磷循环中作用的 研究还刚刚起步,但我们不妨对钲在沉积物与环境之间的迁移进行 具体假设(图 3 ).

沉水植物驱动的水环境钙洜假设为: (1) 沉水植物根系对沉积 物中活性钙的吸收将钙移至菹草茎叶中, 沉水植物的光合作用促使 茎叶吸收水中质子并将钙分泌至水柱中; (2) 沉水植物茎叶分泌的 钲在叶面微域与无机磷结合形成钻磷, 这一作用随着沉水植物光合 作用的加强、水柱 $\mathrm{pH}$ 的升高而加强; (3) 钲磷沉降至沉积物中, 由 于沉水植物能直接向根释放氧气 ${ }^{[45]}$, 有利于根区三价铁生成和沉 淀 ${ }^{[46]}$, 促进钙磷向铁磷的转化, 同时释放出活性钙; (4) 在沉水植物 死亡后, 根际沉积物还原性加强, 沉积物铁磷中 $\mathrm{Fe}$ ( III ) 向 $\mathrm{Fe}$ ( II ) 转 变, 磷又部分得以活化返回水体, 构成环境钙葲作用下水体磷循环; (5) 钙磷沉淀会引起水柱中磷浓度下降, 浮游植物生长得到抑制, 水体透明度增加, 水质得以改善, 水体磷的循环将导致浮游植物暴 发和抑制发生周期性变化. 很显然, 沉水植物驱动的水环境钙洜对

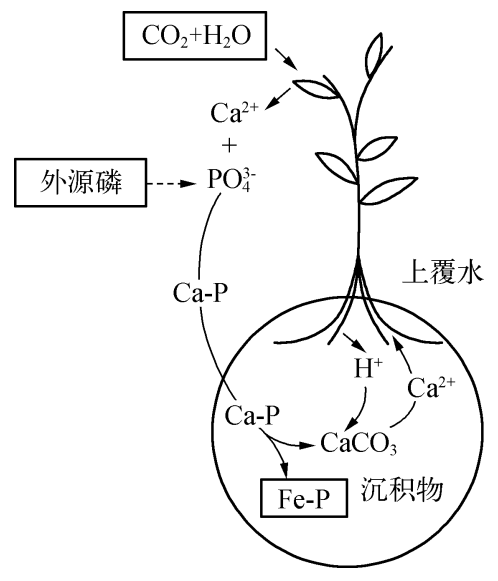

图 3 沉水植物驱动的水环境 钙泵运转模型

Fig. 3 Transport diagram of the water environmental calcium pump driven by submerged macrophytes 沉水植物参与下的水体磷循环起到了举足轻重的作用.

综上所述, 从沉水植物的作用方面研究浅水湖泊水体磷的净化已做了大量工作, 而水体磷循环机理研 究却非常有限, 也少有合适的理论或假说将水体磷的迁移过程与沉水植物的生长联系为一个有机整体. 本 文综合前人对环境、生物体中磷与钲的研究成果和结合本团队的前期部分研究结果将水体磷迁移与沉水植 物吸收、分泌钲相联系, 提出 “水环境钙泵” 之假说, 以指导对沉积物和上覆水中钻迁移在水体磷循环的深人 研究. 根据目前的研究进展, 水环境钙葲的研究重点在于: (1) 开展不同性质沉积物钙分级及钙迁移活性与 沉水植物根系吸收钲特性之间关系的研究, 明确钙在源头迁移速度的决定因素; (2) 开展钙在沉水植物体 内转移的动力学特征及其能量限度研究, 以了解通过沉水植物向上覆水传递钙离子通量和趋势; (3) 研究 沉水植物叶面分泌钲离子的形态学特征, 钙离子在叶面形成碳酸钙的条件及与上覆水磷形成共沉淀的化学 机制和结构表征, 取得钙由沉水植物叶片向水柱释放的关键证据, 形成完整的钙由沉积物经沉水植物向上 覆水迁移, 并与上覆水中较高浓度的碳酸根以及磷形成 $\mathrm{CaCO}_{3}-\mathrm{P}$ 共沉淀理论, 最终揭示沉水植物参与水体 磷地球化学循环的机制.

\section{4 参考文献}

[ 1 ] Conley DJ, Paerl HW, Howarth RW et al. Controlling eutrophication: Nitrogen and phosphorus. Science, 2009, 323 : 1014-1015.

[ 2 ] Srivastava J, Gupta A, Chandra H. Managing water quality with aquatic macrophytes. Rev Environ Sci Biotechnol, 2008, 


\section{7: $255-266$.}

[ 3 ] Gao J, Xiong Z, Zhang J et al. Phosphorus removal from water of eutrophic Lake Donghu by five submerged macrophytes. Desalination, 2009, 242(1/2/3) : 193-204.

[ 4 ] Withers PJA, Jarvie HP. Delivery and cycling of phosphorus in rivers: A review. Sci Total Environ, 2008, 400 : 379-395.

[ 5 ] Gainswin BE, House WA, Leadbetter BSC et al. The effects of sediment size fraction and associated algal biofilms on the kinetics of phosphorus release. Sci Total Environ, 2006, 360 : 142-157.

[ 6 ] Zhang JZ, Huang XL. Relative importance of solid-phase phosphorus and iron on the sorption behavior of sediments. Environ Sci Technol, 2007, 41 : 2789-2795.

[ 7 ] Jarvie HP, Mortimer RJG, Palmer-Felgate EJ et al. Measurement of soluble reactive phosphorus concentration profiles and fluxes in river-bed sediments using DET get probes. J Hydrol, 2008, 350 : 261-273.

[ 8 ] House WA. Geochemical cycling of phosphorus in rivers. Appl Geochem, 2003, 18: 739-748.

[ 9 ] Dodds WK. The role of periphyton in phosphorus retention in shallow freshwater aquatic systems. J Phycol, 2003, 39: 840-849.

[10] McDowell RW. Sediment phosphorus chemistry and microbial biomass along a lowland New Zealand stream. Aquat Geochem, 2003, 9: 19-40.

[11] Siong K, Asaeda T, Fujino T et al. Difference characteristics of phosphorus in Chara and two submerged angiosperm species: implications for phosphorus nutrient cycling in an aquatic ecosystem. Wetl Ecol Manage, 2006, 14 : 505-510.

[12] Jarvie HP, Neal C, Warwick A et al. Phosphorus uptake into algal biofilms in a lowland chalk river. Sci Total Environ, 2002, 282/283 : 353-373.

[13] 徐会玲, 唐智勇, 朱端卫等. 菹草、伊乐藻对沉积物林形态及其上覆水水质的影响. 湖泊科学, 2010, 22 (3): 437-444.

[14] Merico A, Tyrrell T, Wilson PA. Eocene/Oligocene ocean de-acidification linked to Antarctic glaciation by sea-level fall. Nature, 2008, 452 : 979-982.

[15] Schulz HN, Schulz HD. Largesulfur bacteria and the formation of phosphorite. Science, 2005, 307 : 416-418.

[16] Neal C, Jarvie HP, Williams RJ et al. Phosphorus-calcium carbonate saturation relationships in a lowland chalk river impacted by sewage inputs and phosphorus remediation: an assessment of phosphorus self-cleansing mechanisms in natural waters. Sci Total Environ, 2002, 282 : 295-310.

[17] Danen-Louwerse HJ, Lijklema L, Coenraats M. Coprecipitation of phosphate with calcium carbonate in Lake Veluwe. Water Res, 1995, 29: 1781-1785.

[18] Song Y, Hahn HH, Hoffmann E. The effect of carbonate on the precipitation of calcium phosphate. Environ Technol, 2002, 23: 207-215.

[19] McConnaughey TA, LaBaugh JW, Rosenberry DO et al. Carbon budget for a groundwater fed lake: calcification supports summer photosynthesis. Limnol Oceanogr, 1994, 39:1319-1332.

[20] Lee CW, Kwon HB, Jeon HP et al. A new recycling material for removing phosphorus from water. J Clean Prod, 2009, 17: 683-687.

[21] Walpersdorf E, Neumann T, Stüuben D. Efficiency of natural calcite precipitation compared to lake marl application used for water quality improvement in an eutrophic lake. Appl Geochem, 2004, 19: 1687-1698.

[22] Song Y, Weidler PG, Berg U et al. Calcite-seeded crystallization of calcium phosphate for phosphorus recovery. Chemosphere, 2006, 63: 236-243.

[23] Diaz J, Ingall E, Benitez-Nelson C et al. Marine polyphosphate: A key player in geologic phosphorus sequestration. Science, 2008, 320: 652-655.

[24] 倪玲珊. 蕰草对长江中游湖泊沉积物及上覆水间钲、磷循环的影响 [ 学位论文]. 武汉: 华中农业大学,2010.

[25] Bajnóczi B, Kovács-Kis V. Origin of pedogenic needle-fiber calcite revealed by micromorphology and stable isotope composition- a case study of a Quaternary paleosol from Hungary. Chemie der Erde, 2006, 66 : 203-212.

［26］赵海超,赵海香, 王圣瑞等. 沉水植物对沉积物及土壤垂向各形态无机磷的影响. 生态环境, 2008,17(1):74-80.

[27] Sigman DM, Boyle EA. Glacial/interglacial variations in atmospheric carbon dioxide. Nature, 2000, 407: 859-869.

[28] Kintisch E. Should oceanographers pump iron? Science, 2007, 318: 1368-1370.

[29] Hepler PK. Calcium, a central regulator of plant growth and development. Plant Cell, 2005, 17: 2142-2155. 
[30] White PJ. The pathways of calcium movement to the xylem. J Exp Bot, 2001, 52: 891-899.

[31] Deng D, Wu S, Wu F et al. Effects of root anatomy and Fe plaque on arsenic uptake by rice seedlings grown in solution culture. Environ Pollut, 2010, 158 : 2589-2595.

[32] Connell EL, Colmer TD, Walker DI. Radial oxygen loss from intact roots of Halophila ovalis as a function of distance behind the root tip and shoot illumination. Aquat Bot, 1999, 63: 219-228.

[33] Cholewa E, Peterson CA. Evidence for symplastic involvement in the radial movement of calcium in onion roots. Plant Physiol, 2004, 134: 1793-1802.

[34] Yang HQ, Jie YL. Uptake and transport of calcium in plants. J Plant Physiol Mol Biol, 2005, 31: $227-234$.

[35] Ramadan T. Ecophysiology of salt excretion in the xero-halophyte Reaumuria hirtella. New Phytol, 1998,139 : $273-281$.

[36] 刘志华,时丽冉,赵可夫. 獐茅盐腺形态结构及其泌盐性. 植物生理与分子生物学学报,2006,32:420-426.

[37] White PJ, Broadley MR. Calcium in plants. Ann Bot, 2003, 92 : 487-511.

[38] Olesen C, Picard M, Winther AL et al. The structural basis of calcium transport by the calcium pump. Nature, 2007, 450: 1036-1042.

[39] Andersen JP, Jorgensen PL, Moller JV. Direct demonstration of structural changes in soluble, monomeric Ca ${ }^{2+}-\mathrm{ATPase}$ associated with $\mathrm{Ca}^{2+}$ release during the transport cycle. Proc Natl Acad Sci USA, 1985, 82 : 4573-4577.

[40] Hirschi KD. Vacuolar $\mathrm{H}^{+} / \mathrm{Ca}^{2+}$ transport, who's directing the traffic? Trends Plant Sci, 2001, 6: 100-104.

[41] Pottosin II, Schönknecht G. Vacuolar calcium channels. J Exp Bot, 2007, 58 : 1559-1569.

[42] Hu X, Xiang C, Cao L et al. A mathematical model for ATP-mediated calcium dynamics in vascular endothelial cells induced by fluid shear stress. Appl Math Mech-Engl, 2008, 29: 1291-1298.

[43 ] Barat R, Montoya T, Borrás L et al. Interactions between calcium precipitation and the polyphosphate-accumulating bacteria metabolism. Water Res, 2008, 42 : 3415-3424.

[44] 杨洪强, 张连忠, 戚金亮等. 苹果砧木根系钲素吸收动力学研究. 园艺学报,2003,30(3):253-257.

[45] Karjalaincn H, Stefansdottir G, Tuominen L et al. Do submersed plants enhance microbial activity in sediment? Aquat Bot, 2001, 69(1): 1-13.

[46] Hupfer M, Dollan A. Immobilisation of phosphorous by ion-coated roots of submerged macrophytes. Hydrobiologia, 2003, 506/509: 635-640. 